\title{
ON A PROBLEM OF N. ARONSZAJN AND AN AXIOM OF R. L. MOORE*
}

\section{BY LEO ZIPPIN $\dagger$}

In an interesting paper N. Aronszajn $\ddagger$ raises the question whether every space belonging to a class of topologic spaces which he introduces $\S$ is necessarily an absolute $G_{\delta}$-set; that is, one which in every metric space $M$ containing it may be expressed as the product of a countable set of sets open in $M$. In this, the first part of the paper gives an affirmative answer. The class of spaces $R$ is given, in effect, by the following axiom: a topologic space $R$ belongs to the class $R$ if and only if there exists an infinite sequence of classes $\Pi_{R}^{n}(R)$ for $n=1,2, \cdots$, such that

(1) for every natural number $n, R$ is generated by $\Pi_{R}{ }^{n}(R)$;

(2) with every sequence of sets $\left\{\bar{U}_{n}\right\}$, where $U_{n} \supset U_{n+1}$, there is associated a point $p$ of space such that $p=\Pi_{i=1}^{\infty} \bar{U}_{i}$ and this sequence converges to $p$.

The necessary definitions are these: a class $\Pi_{R}(R)$ is any class of point sets of $R$ which are open in $R$ and whose sum covers $R$. Such a class generates $R$ if, further, given any point $p$ of $R$ and any open set $U$ containing $p$, then there is an element $V$ of $\Pi_{R}(R)$ which contains $p$ and is contained in $U$. A set of sets converges to a point if every open set containing the point contains all but a finite number of the given sets.

We suppose then that $R$ is a space as defined above and is a subset of a metrizable space $M$. Then whatever metric be associated with $M$ (preserving its topology) this is induced on $R$ as a subspace (preserving its topology) and we may speak of the diameters of the elements of any of the generating classes. Leit $U$ be an element of one of these classes whose diameter is finite

* Presented to the Society, December 31, 1930.

$\dagger$ National Research Fellow.

$\ddagger$ N. Aronszajn, Über die Bogenverknüpfung in topologischen Räumen, Fundamenta Mathematicae, vol. 15 (1930), pp. 228-241.Compare the first paragraph of page 229.

$\S$ Part of this paper is concerned with the relation of these spaces to spaces defined by R. L. Moore considerably earlier. 
(the restriction is convenient) and suppose its diameter, $\epsilon$, not zero. For each point $k$ of $U$ there is in $M$ a spherical neighborhood of $k$ of diameter at most $\epsilon / 3$ which contains no point of $R$ not in $U$, since $U$ is open in $R$. The sum of all such spherical neighborhoods for every point $k$ of $U$ gives a set $U^{*}$ which is open in $M$, of diameter less than $2 \epsilon$, and such that $U=R \cdot U^{*}$. If $\epsilon$ is zero, and $U$ reduces to a point $k$ of $R$, we shall suppose $U$ indefinitely repeated and associate with it an infinite set of spherical neighborhoods $U^{*}$ of diameters converging to zero such that $R \cdot U^{*}=k$. We shall call $U^{*}$ the extended element of that class of which $U$ is an element. If $U$, above, is vacuous, it is not relevant to the definition of limit points in $R$, and is ignored.

Let $F_{n, m}$ be the set of all points of $M$ which belong to no extended element of the nth generating class which is of diameter less than $1 / m$. This is seen to be closed in $M$ since the extended elements are open there. Then

$$
F=\sum_{n=1}^{\infty} \sum_{m=1}^{\infty} F_{n, m}
$$

is the sum of a countable set of closed sets, and $G=M-F$ is a $G_{\delta}$-subset relative to $M$. If, now, $x$ is any point of $R$ and $m$ and $n$ are any preassigned natural numbers, let $U_{x}^{*}$ be a spherical neighborhood of $x$ in $M$ of radius less than $1 /(4 m)$. Since $U_{x}=R \cdot U_{x}^{*}$ is open in $R$ there is an element $U$ of the $n$th generating class containing $x$ and contained in $U_{x}$, and therefore of diameter less than $1 /(2 m)$. Consequently this has a corresponding extended element $U^{*}$ of diameter less than $1 / m$, and $U^{*}$ contains $x$ which cannot, therefore, belong to $F_{n, m}$. Since this is true for every point of $R$, and every $n$ and $m, R$ must belong to $G$. Suppose, on the other hand, that $y$ is a point of $G$; then it belongs to no $F_{n, m}$ and is contained in an extended element of any preassigned generating class which is, moreover, of diameter less than any preassigned positive number, not zero. Then $y$ belongs to an extended element $U_{1}^{*}$ which is of diameter less than 1 . There is a spherical neighborhood $U_{y}^{*}$ of $y$ in $M$ such that $U_{y}^{*} \subset U_{1}^{*}$. Let $1 / m_{1}$ be less than half the radius of $U_{y}^{*} ; y$ belongs to an extended element $U_{2}^{*}$ of the second generating class which is of diameter less than $1 / m_{1}$, and $U_{2}^{*} \supset U_{1}^{*}$. Continuing inductively, we see that there exists a sequence of extended elements, $U_{1}^{*}, U_{2}^{*}, \cdots$, $U_{n}^{*}, \cdots$, each containing $y$, of diameters converging to zero, 
each a subset of its predecessor, and each an extended element of the generating class of corresponding number. But $U_{n}=R \cdot U_{n}^{*} \supset R \cdot U_{n+1}^{*}=U_{n+1}$. Then, by the second condition of the axiom, the set of elements $U_{n}$ must converge to a point $y^{\prime}$ of $R$, and this must be identical with $y$. Then every point of $G$ is a point of $R$, and it is shown that $R$ is a $G_{\delta}$-subset of $M$.

We shall prove that in metrizable topologic spaces the axiom of $\mathrm{N}$. Aronszajn is equivalent to the following earlier axiom of $\mathrm{R}$. L. Moore. $\dagger$ For every natural number $n, G_{1}, G_{2}, \cdots, G_{n}, \cdots$ is a collection of regions such that (1) for every $n, G_{n}$ covers space, (2) if $R$ is a region and $p$ and $q$ are points of $R$, there is an integer $d$ such that if $n \geqq d$ and $R_{n}$ is a region of $G_{n}$ containing $p$, then $\bar{R}_{n} \subset R-q,(3)$ if $R_{1}, R_{2}, \cdots$ is a set of regions such that for each $n, R_{1}, R_{2}, \cdots, R_{n}$ have a common point, then there is a point $p$ such that $p \subset \Pi_{i=1}^{\infty} \bar{R}_{i}$.

Let $R$ be a space satisfying the axiom of Aronszajn and, moreover, metric. We shall define a sequence of generating classes, $G_{1}, G_{2}, \cdots, G_{n}, \cdots$, composed of elements of the corresponding index. It is to be borne in mind that since every spherical neighborhood $U_{p}$ of a point $p$ of $R$ is open in $R$, there exists in whatever generating class we wish an element containing $p$ and contained in $U_{p}$. Let $G_{1}$ be the set of all elements of $\Pi_{R}{ }^{1}(R)$ of diameter less than 1. For each point $p$ of $R$ there is some element $U_{p}^{1}$ of $G_{1}$ which contains it. There is a lower limit $\epsilon_{p}$, distinct from zero, to the distances from the point $p$ to the points of $R-U_{p}^{1}$. With $p$ we associate all those elements of $\Pi_{R}{ }^{2}(R)$ which contain $p$ and whose diameter is less than $\epsilon_{p} / 4$. The set of all such elements, corresponding to each point of $R$ for some element of $G_{1}$ arbitrarily associated with it, as above, is the set $G_{2}$. Analogously, for every $n$ we form $G_{n}$ from among the elements of $\Pi_{R^{n}}{ }^{n}(R)$, with respect to the elements of $G_{n-1}$. That each $G_{n}$ is a generating class for $R$, and that in their totality they form a system equivalent to the original (define the same topologic space and have the properties of the original system of elements) is clear. Every element of $G_{n}$, for every $n$, we shall

$\dagger$ With this axiom and the consequences Moore deduced from it, Aronszajn appears not to be acquainted. Abstract, this Bulletin, vol. 33 (1927), p. 141. A slightly different version of this axiom was presented by R. L. Moore at the Boulder Colloquium Lectures in August of 1929, but the two are readily shown to be equivalent. 
call a region (which is an undefined concept in Moore's axiom) and these only shall be regions.

The first condition of Moore's axiom is obviously satisfied by this sequence of regions, and the second by virtue of the fact that a region of $G_{n}$ is of diameter at most $1 / 4^{n-1}$. Suppose now that we have a set of regions $R_{1}, R_{2}, \cdots$, satisfying the requirements of the third condition of Moore's axiom. Since $R_{2}$ is a region of $G_{2}$, by the construction of $G_{2}$, there exists a point $p$ of $R_{2}$ and a region $U_{p}^{1}$ of $G_{1}$ such that*

$$
\delta\left(R_{2}\right)<\epsilon_{p} / 4=\rho\left(p, R-U_{p}^{1}\right),
$$

and $\delta\left(U_{p}^{1}\right) \geqq \epsilon_{p}$. It is seen that $\rho\left(R_{2}, R-U_{p}^{1}\right) \geqq 3 \epsilon_{p} / 4$. Let $K_{2}$ be any region of $G_{2}$ which contains $R_{3}$ and let us suppose that $K_{2}$ is not contained in $U_{p}^{1}$. Since $K_{2} \supset R_{3} \supset R_{3} \cdot R_{2} \neq 0$, and also $K_{2} \cdot\left(R-U_{p}^{1}\right) \neq 0$, it follows that $\delta\left(K_{2}\right) \geqq 3 \epsilon_{p} / 4$. Then there is a point $q$ of $K_{2}$ and a region $U_{q}^{1}$ of $G_{1}$ containing $K_{2}$, such that $\delta\left(K_{2}\right)<\epsilon_{q} / 4$, and it follows that $\delta\left(U_{q}^{1}\right)>3 \epsilon_{q}$. Since the set of diameters of regions of $G_{1}$ is limited above, this process of selecting successive regions of $G_{1}$ must terminate with a region $P_{1}$ of $G_{1}$ with the following properties: there is an integer $n_{1}$ such that if $m \geqq n_{1}, P_{1} \supset R_{m}$; and further if $Q_{2}$ is any region of $G_{2}$ containing $R_{m}$, then $Q_{2} \subset P_{1}$. It is possible, inductively, to determine a set of regions, $P_{1}, P_{2}, \cdots, P_{n}, \cdots$, and a set of integers $n_{1}, n_{2}, \cdots$, such that (1) $P_{n}$ is a region of $G_{n}$, (2) $P_{n} \supset P_{n+1}$, (3) if $m \geqq n_{k}$, then $R_{m} \subset P_{k}$. From the first two of these properties it follows (by the second condition of Aronszajn) that the regions (elements) $P_{n}$ converge to a point $p$ of $R$. Then for every region $P \supset p$, there is an integer $k$ such that if $m \geqq k, P \supset P_{m}$. By the third property above, $P \supset R_{j}$ where $j \geqq n_{m}$. But since $R_{i} \cdot R_{j} \neq 0$, where $i=1,2, \cdots, j$, it follows that $P$ contains a point of $R_{i}, i=1,2,3, \cdots$, and $p \subset \bar{R}_{i}$. Then we have shown that $R$ satisfies the axiom of Moore.

We may define a class of topologic spaces, in a sense the most general, in which an Aronszajn space is necessarily a Moore space, by adjoining to conditions (1) and (2) of Moore's axiom a third to the effect that to a set of regions as described in Moore's axiom may be associated a set $P_{1}, P_{2}, \cdots$, with properties as above. We have seen that all metric spaces are in-

\footnotetext{
* Read: the diameter of $R_{2}$ less than lower limit of distances of $p$ to points of $R-U_{p}$.
} 
cluded among these and it may be shown that this class comprises all Moore spaces, and these include examples of nonmetric spaces. And it is true, further, that a space of Moore is a space of Aronszajn always. But the author's proofs of these statements he finds to be implicit in various arguments which R. L. Moore has already used (in the pages of his work to appear, in the near future, in the regular Colloquium Series) and in consequence no proof will be given.

The following example of a space satisfying the axiom of Aronszajn (given by him in his paper, page 239) will be used to show that his axiom is more general than Moore's. Let $T$ be the set of all ordinal numbers of the first and second class, where for $n=1,2,3, \cdots$, the $n$th neighborhood of $a, U_{n}(a)=a$ if $a$ is an isolated ordinal, while if $a$ is a limit-ordinal $U_{n}(a)$ is a set of numbers $z$ such that $b_{n}<z \leqq a$, wher $\lim _{n \rightarrow \infty}\left(b_{n}\right)=a$. Now, however region be defined or this space in accordance with Moore's axiom, for every limit number $p$ there must occur in one of the covering sets a region $R_{p}$ which contains none of the successors of $p$, and $R_{p}$ must contain infinitely many of the predecessors of $p$; in particular, one other number $q$ Then we may associate with $p$ a number $n_{p}$ such that every region of the $n_{p}$ th covering set which contains $p$ is contained in $R_{p}$, and contains therefore no successor to $p$. But the set of limit-numbers being uncountable, to uncountably many (in particular to a countable, infinite subset) must correspond the same integer $N$. Then there is an infinite set $\left(x_{n}\right)$ of these numbers, with a limit number $x$ which succeeds all of them. A region of the $(N+1)$ th covering set containing $x$ must contain at least one point of $\left(x_{n}\right)$ contradicting the choice of $N$. From the self-compactness of the space $T$, the third condition of Moore's axiom is necessarily fulfilled; but the question whether the fundamental difference between the axioms is constituted by the "uniformity of regularity" which inheres in Moore's second condition (contradicted in the space $T$ ) is not settled.

The relation between the axioms is the more challenging since they were fashioned with the same end in view and Moore and Aronszajn drew from them, for their respective spaces, the same impressive consequence.

The University of Texas 